\title{
Willy Astors Promi-WG: Zur phonetischen Signalisierung von Wortspielen
}

\section{Einleitung}

Willy Astor gehört zweifellos zu den deutschsprachigen Kabarettisten mit einer besonders ausgeprägten Sensibilität für Sprache. Er hat sichtlich Freude am filigranen Spiel mit Sprachlautung. Ein Beispiel für seinen virtuosen Umgang mit Lautähnlichkeiten ist der Teil seines Programms, der unter dem Rubrum „PromiWG“ bekannt geworden ist. Der Beitrag besteht aus dem lautlichen Spiel mit den Namen von 51 Prominenten, die teils lautlich übernommen, aber rekontextualisiert und teils lautlich reinterpretiert werden. Ein Beispiel für ersteren Fall ist Claus Theo Gärtner, dessen Vorname beibehalten und Nachname zur Berufsbezeichnung umgedeutet wird („Der Claus Theo war unser Gärtner“). Der zweite Fall wird z. B. durch Morgan Freeman repräsentiert, dessen Name als „morgen früh mähn“ uminterpretiert wird. ${ }^{1}$

Dieser Sketch wird im Folgenden unter zwei Aspekten analysiert. Zum einen geht es darum, welche Namensbestandteile (Vorname, Nachname, Vor- und Nachname) in welcher Weise „bespielt“ werden, zum zweiten, was aus phonetischer Sicht mit den Namen passiert, d. h. konkret, welche Mechanismen bei der Umdeutung der Namen zur Anwendung kommen und wie stark die Namen verfremdet werden können, ohne dass die gewünschte Publikumsreaktion ausbleibt.

1 Im Anhang findet sich eine komplette Verschriftung des Sketches. Die Darstellung stützt sich auf eine in Youtube veröffentlichte Fassung (vgl. die Quellenangabe im Anhang). Somit können sämtliche Analysen streng genommen nur für diese Vorstellung Gültigkeit beanspruchen. Andererseits sind die Ergebnisse so eindeutig, dass angenommen werden darf, dass sie sich so oder ähnlich auch bei anderen Auftritten finden. 


\section{Wortspiel-Strategien}

\subsection{Welche Namensbestandteile werden bespielt?}

Drei verschiedene Strategien im Umgang mit den Namen der Prominenten lassen sich unterscheiden:

(a) Nur der Nachname wird in das Wortspiel einbezogen. In diesen Fällen behält der Vorname seine lautliche Form und seine Funktion, d. h. er wird als Name verwendet. Er dient dabei häufig als kataphorischer Hinweisreiz (cue) für die Re-Interpretation des Nachnamens und erleichtert diese durch die Schaffung von Kontext. Beispiele sind Bruno Ganz und Günther Maria Halmer. Diese Konstellation ist mit 38 von 51 Fällen die häufigste.

(b) Nur der Vorname wird in das Wortspiel einbezogen. In diesen Fällen behält der Nachname seine lautliche Form und seine Funktion, d. h. er wird als Name verwendet. Es fällt allerdings auf, dass dabei - anders als in (a) - der Nachname vor dem Vornamen eingeführt wird, so dass er wiederum als Hinweisreiz (cue) für die Interpretation des Vornamens dienen kann. Beispiele sind Gwyneth Paltrow und Horst Seehofer. Insgesamt ist diese Konstellation in dem untersuchten Sketch in drei von 51 Fällen gegeben und damit am seltensten.

(c) Vor- und Nachname werden in das Wortspiel einbezogen. Ein Beispiel ist Anton, i hob kins 'Anthony Hopkins' oder schon penn 'Sean Penn'. In diesen Fällen liefert weder der Vor- noch der Nachname Kontextinformation, so dass die Dekodierung höhere Ansprüche an das Publikum stellt, als dies bei kontextuell vorbereiteten Re-Interpretationen der Namen der Fall ist. Dies geschieht bei zehn der 51 Namen.

Anhand der Publikumsreaktionen soll nun untersucht werden, ob sich tatsächlich Hinweise auf einen erhöhten Dekodierungsaufwand bei denjenigen Sketches finden, die auf Vor- und Nachnamen zurückgreifen.

\subsection{Was geschieht mit den Namensbestandteilen?}

Insbesondere unter kognitionspsychologischem Aspekt ist jedoch auch die linguistisch-funktionale Ebene zu bedenken. Dabei geht es um die Frage, ob der Nachname als Substantiv erhalten bleibt, oder ob die Wortart verändert wird. Es liegen zahlreiche Studien vor, aus denen hervorgeht, dass Personennamen anders verarbeitet werden als Substantive (vgl. z. B. Brédart, Brennen und Valen- 
tine 1997; Hollis und Valentine 2001; Valentine, Moore und Brédart 1995; Proverbio et al. 2009, Semenza 2009). Letzterer fasst den Forschungsstand wie folgt zusammen (Semenza 2009: 364-365):

[...] common names and proper names are shown to follow different processing pathways. These pathways are neurally distinct and differently sensitive to brain damage. However, their exact location, depending on specific tasks, is still partly unknown.

The pathway followed by proper names seems to require additional cognitive and organic resources in different tasks, e.g. in lexical retrieval and in assigning the correct syntactical context. Functionally, these pathways are separate at different levels including the access to phonological forms.

Man könnte danach argumentieren, dass die Veränderung der Wortart eine erhöhte Anforderung an die kognitive Verarbeitung stellt, zumal ein Vermittlungsschritt zwischen Namen und (homophonem) Substantiv vollzogen werden muss. Hier werden drei Fälle unterschieden:

(a) Ein Namensbestandteil bleibt als Name erhalten; der zweite wird als Substantiv interpretiert (z. B. Wencke Myhre, Cameron Diaz).

(b) Ein Namensbestandteil bleibt als Name erhalten; der zweite verändert jedoch die Wortart (z. B. Bruno Ganz, Jan-Josef Liefers, Ben Stiller).

(c) Ein Namensbestandteil wird in mehrere Lexeme aufgespalten (Robert Redford, Morgan Freeman).

Der erstgenannte Fall tritt insgesamt 24 Mal auf, der zweite ist 18 Mal zu beobachten, und der dritte findet sich 14 Mal. Hier könnte man vermuten, dass die Umdeutung eines Namensbestandteils als Substantiv weniger komplexe Verarbeitungsprozesse erfordert als die Umdeutung zu einer anderen Wortart oder gar die lexikalische Reorganisation.

\subsection{Wie ausgeprägt ist die phonetisch-phonologische Veränderung?}

Eine weitere Frage, die Einfluss auf die Dekodierungsgeschwindigkeit haben könnte, ist der Grad der phonetischen Veränderung gegenüber dem Original. Dabei sollen hier vier Fälle unterschieden werden, weil sie möglicherweise den hörerseitigen Verarbeitungsprozess beeinflussen:

(a) Komplette Homophonie, d. h. Vor- und Nachname sind phonetisch und phonologisch auf segmenteller und suprasegmenteller Ebene vollkommen identisch mit der Ziel-Lautung, und die Reinterpretation geschieht durch Rekontextualisierung. Ein Beispiel dafür ist Wencke Myhre. 
(b) Komplette Homophonie auf segmenteller Ebene, wobei die prosodische Ebene Unterschiede aufweist. Ein Beispiel ist Harrison Ford, der im Sketch abweichend als ['hexison fo:t] betont wird.

(c) Quasi-Homophonie (Homoiophonie; cf. Braun 2018), bei der die phonologische Struktur erhalten bleibt, sich aber die phonetische Realisierung unterscheidet. Ein Beispiel ist (ich) gwinn es ['gvinəs] im Zusammenhang mit Gwyneth ['gwinə日] (Paltrow), wobei die im Englischen bestehenden phonologischen Unterschiede zwischen dentalen und alveolaren Frikativen einerseits und zwischen labiovelaren Approximanten und labiodentalen Frikativen andererseits im Deutschen nicht phonologisch sind und daher der dentale Frikativ / $\theta$ / problemlos durch artikulatorisch eng verwandtes [s] und der labiovelare Approximant [w] durch [v] ersetzt werden können (Braun 2018).

(d) Größere phonologische Unterschiede zwischen tatsächlichen Namen und rekontextualisierter Form (Beispiele sind André Diarrhoe: 'André Rieu' und Morgan Freeman: engl. [mว:gən 'fui:mən] vs. dt. [məegən 'fxy: me:n] $]^{2}$

\section{Pilotstudie}

Eine ausführliche empirische Untersuchung der oben aufgeworfenen Fragen würde den Rahmen dieses Beitrags sprengen. Daher wird hier zunächst eine explorative Studie vorgestellt, die erproben soll, inwieweit die vorgeschlagenen Parameter überhaupt untersuchbar sind, und ob erste Ergebnisse weitere Untersuchungen sinnvoll erscheinen lassen.

\subsection{Messgrößen}

Als Maß für den Verarbeitungsaufwand seitens der Zuhörer wurden die Zeitspanne zwischen dem Ende der Namensäußerung bzw. der Pointe und dem Intensitätsmaximum ${ }^{3}$ des Lachens (Latenzzeit) sowie die Dauer des Lachens gewählt. Erstere stellt ein Maß dafür dar, wie schnell ein Witz „zündet“. Letztere ist dem Umstand geschuldet, dass Zuhörer, die den Witz erst später begreifen, auch später reagieren, wodurch die Gesamtdauer der Lacher ansteigen sollte. Aus Gründen der Vergleichbarkeit wurden dabei nur diejenigen Fälle berücksich-

2 Offenbar um das Verständnis zu erleichtern, spricht Astor das /r/ auch im Deutschen als alveolaren Approximanten $[x]$ aus.

3 Dies entspricht dem auditiven Lautstärkemaximum. 
tigt, bei denen der Name zugleich die Pointe bildet ${ }^{4}$, und es wurde das Zeitintervall zwischen dem Ende der Namensnennung und der maximalen Intensität der Lacher gemessen.

Die durchschnittliche Intensität der Lacher hingegen sollte sich nicht signifikant von der aus den übrigen Fällen unterscheiden, weil das Lachen sich auf einen längeren Zeitraum verteilt und folglich keinen deutlichen Intensitätsgipfel herausbildet.

\subsection{Hypothesen}

Für die empirische Untersuchung wurden daher die folgenden Hypothesen aufgestellt:

H1 Je komplexer die Dekodierungsaufgabe, umso länger die Latenzzeit. Dies würde bedeuten, dass die Latenzzeit signifikant länger wird, wenn sowohl der Vor- als auch der Nachname in das Wortspiel einbezogen werden.

H2 Je komplexer die Dekodierungsaufgabe, umso länger die Dauer der Publikumsreaktion. Es wird erwartet, dass die Dauer der Lacher im Publikum signifikant länger wird, wenn sowohl Vor- als auch Nachname in das Wortspiel integriert werden.

H3 Die durchschnittliche Intensität in solchen Fällen, in denen beide Namensbestandteile in das Wortspiel integriert sind, unterscheidet sich nicht signifikant von der in denjenigen Fällen, in denen nur ein Namensbestandteil für das Wortspiel Verwendung findet.

H4 Es wird erwartet, dass die Latenzzeit signifikant länger wird, wenn die Wortart verändert wird bzw. die Namen relexikalisiert werden.

H5 Es wird erwartet, dass die Dauer der Lacher im Publikum signifikant länger wird, wenn die Wortart verändert wird bzw. die Namen relexikalisiert werden.

H6 Die durchschnittliche Intensität in solchen Fällen, in denen der Nachname Substantiv bleibt, unterscheidet sich nicht signifikant von der in denjenigen Fällen, in denen die Wortart verändert wird bzw. die Namen relexikalisiert werden.

4 Dies ist bei 46 von 51 Namen der Fall. Es kommt jedoch auch vereinzelt vor, dass nach der Nennung des Namens (Pointe) noch weitergesprochen wird. Ein Beispiel ist Dass der Jürgen an Vogel hat, war klar. Diese Konstellation besteht lediglich fünf Mal. Da in solchen Fällen nicht klar entscheidbar war, ob der Beginn der Latenzzeit nach dem Namen oder am Äußerungsende angesetzt werden musste, wurden sie nicht in die Untersuchung einbezogen. 


\subsection{Ergebnisse}

Die Hypothesen H1 bis H3 wurden unter Verwendung des Software-Pakets Systat 10 anhand von t-Tests (zweiseitig; separate Varianzen) untersucht. Die Hypothesen $\mathrm{H} 4$ bis $\mathrm{H} 6$ wurden mittels derselben Software anhand von Varianzanalysen (ANOVA) überprüft. Dabei kam ein Tukey post-hoc Test zur Anwendung, um paarweise Signifikanzen zu prüfen. Das Signifikanzniveau wurde auf 5\% festgelegt.

Die Mittelwertsdifferenz hinsichtlich der Latenz in Höhe von $361 \mathrm{~ms}$ erweist sich als hochsignifikant zwischen solchen Fällen, in denen ein Namensbestandteil in seiner Funktion erhalten bleibt, während der andere eine Re-Interpretation erfährt, und solchen, in denen beide Namensbestandteile Teil des Sketches sind und im Zuge dessen verändert werden (t-Test zweiseitig, separate Varianzen; $\mathrm{p}=$ 0,004; vgl. Abb. 1). Ein Beispiel für den ersteren Fall ist Bruno Ganz; ein Beispiel für den zweiten Fall ist Alice Schwarzer.

Hypothese \#1 kann somit als bestätigt gelten.

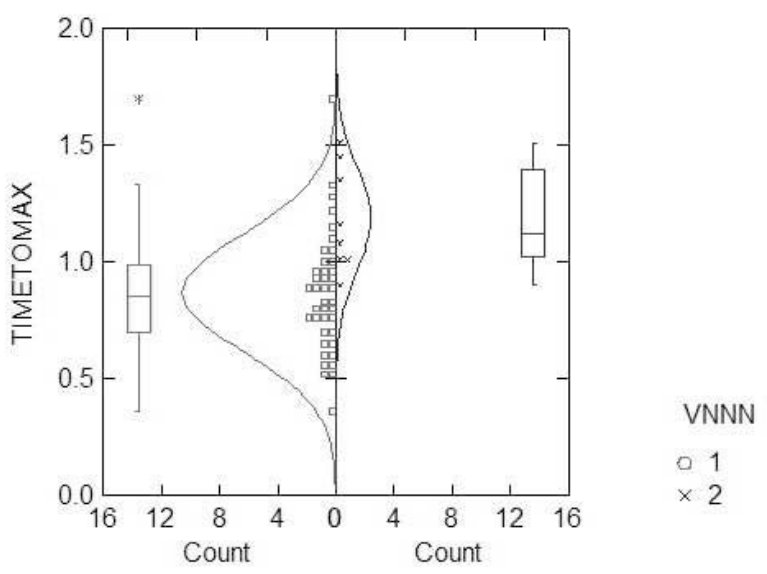

Abb. 1: Latenzzeit bis zum Erreichen des Intensitätsmaximums in Abhängigkeit vom betroffenen Namensbestandteil ( $1=$ Vor- oder Nachname; 2 = Vor- und Nachname)

Die Dauer der Lacher in Abhängigkeit davon, ob beide Namensbestandteile durch das Wortspiel erfasst werden oder nicht, weist eine deutliche Tendenz in die erwartete Richtung auf (längere Dauer bei Veränderung beider Namens- 
bestandteile). Der Unterschied erweist sich allerdings als nicht signifikant (t-Test einseitig; $p=0,180$, vgl. Abb. 2).

Hypothese \#2 muss somit verworfen werden.

Gleichermaßen erweist sich die mittlere Intensität der Lacher nicht als distinktiv zwischen beiden Fallgruppen (t-Test zweiseitig, separate Varianzen; $\mathrm{p}=0,481$.

Hypothese \#3 kann somit als bestätigt gelten.

Die Latenzzeit bis zum Erreichen des Intensitätsmaximums nimmt mit dem Grad der Wortartveränderung tendenziell zu (vgl. Abb. 3), aber der Unterschied erreicht nicht das erforderliche Signifikanzniveau ( $p=0,288$ zwischen Adjektiv und Substantiv; Tukey post-hoc testing).

Hypothese \#4 muss somit verworfen werden.

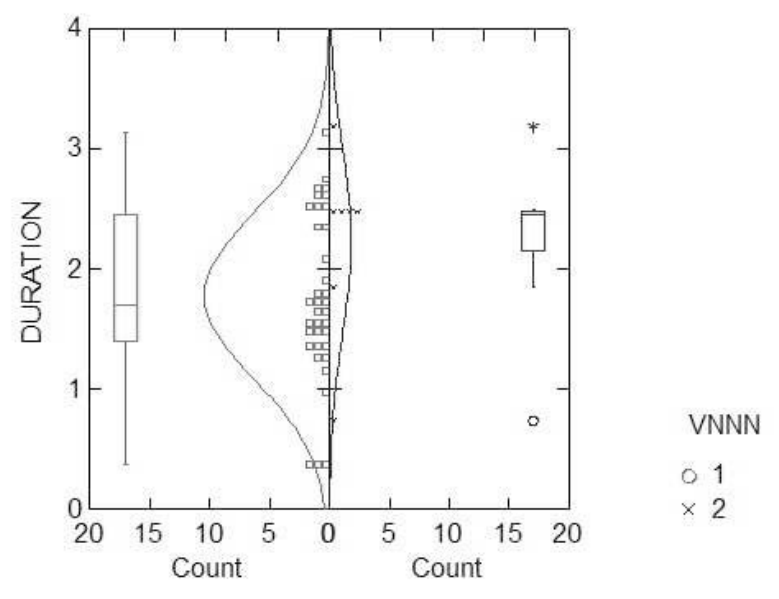

Abb. 2: Dauer der Lacher in Abhängigkeit vom betroffenen Namensbestandteil ( $1=$ Vor- oder Nachname; 2 = Vor- und Nachname)

Die Dauer der Lacher wächst zwar mit der Komplexität der Dekodierungsaufgabe (vgl. Abb.4), aber der Unterschied erreicht nicht das angestrebte Signifikanzniveau ( $\mathrm{p}=0,134$ zwischen Adjektiv und Umformung in mehrere Lexeme; Tukey post-hoc testing).

Hypothese \#5 muss somit verworfen werden. 


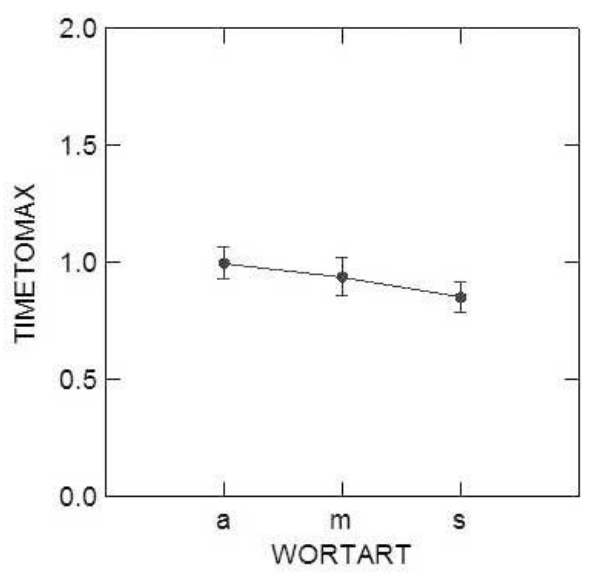

Abb. 3: Latenzzeit bis zum Erreichen des Intensitätsmaximums in Abhängigkeit von der lexikalischen Transformation ( $\mathrm{s}=$ Substantiv; $\mathrm{a}=$ Adjektiv; $\mathrm{m}=$ mehrere Lexeme)

Hinsichtlich der mittleren Intensität der Lacher findet sich kein signifikanter Unterschied in Abhängigkeit von der Art der lexikalischen Transformation ( $p=0,210$ zwischen Substantiv und Aufspaltung in mehrere Lexeme; Tukey posthoc testing).

Hypothese \#6 kann somit bestätigt werden.

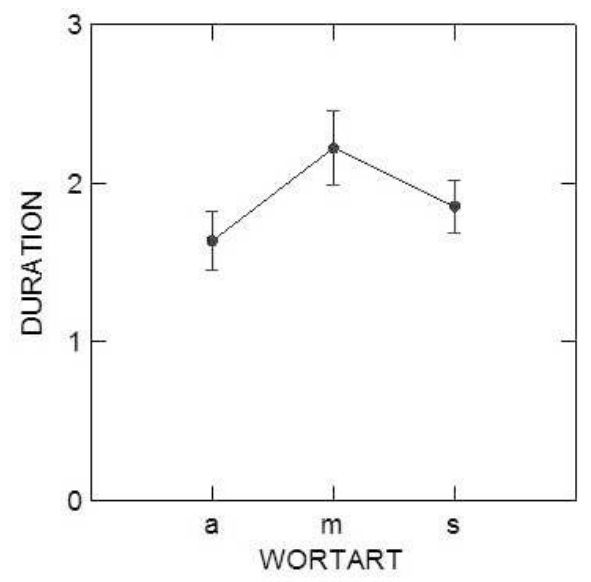

Abb. 4: Dauer der Lacher in Abhängigkeit von der lexikalischen Transformation (s = Substantiv; $\mathrm{a}=$ Adjektiv; $\mathrm{m}=$ mehrere Lexeme) 
Was die phonetisch-phonologischen Veränderungen betrifft, so erfolgt die Auswertung qualitativ. Tab. 1 bietet einen Überblick über die Verteilung dieser Varianten in dem Sketch. Die Zahlen machen deutlich, dass je nach lexikalischer Umdeutung unterschiedliche phonetische Mechanismen präferiert werden.

Tab. 1: Lexikalische und phonetische Klassifikation der Wortspiele

\begin{tabular}{lrrrr}
\hline $\begin{array}{l}\text { Phonet. Form } \\
\text { Lexikalische }\end{array}$ & Homophonie & $\begin{array}{r}\text { Homophonie } \\
\text { (nur segmentell) }\end{array}$ & Homoiophonie & Heterophonie \\
\hline $\begin{array}{l}\text { Substansformation } \\
\begin{array}{l}\text { Andere Wortart } \\
(\mathrm{N}=18)\end{array}\end{array}$ & 17 & - & 5 & 2 \\
$\begin{array}{l}\text { Aufsplitterung in } \\
\text { mehrere Lexeme }\end{array}$ & 3 & 2 & 4 & 1 \\
$(\mathrm{~N}=14)^{5}$ & 1 & & 9 & 2 \\
\hline
\end{tabular}

Insgesamt wird am häufigsten mit der Substitution von (Nach-)Namen durch homophone Substantive gearbeitet (vgl. beispielsweise Claus Theo Gärtner oder Wencke Myhre). Dies kann als die aus der Perspektive des Autors „sicherste“ Variante gelten, da sie keine lautliche Re-Interpretation durch das Publikum erfordert. Andererseits muss natürlich die Ambiguität der Formulierung erkannt werden. Ein wesentliches Hemmnis bei der erfolgreichen Dekodierung bildet die mangelnde Vertrautheit mit dem jeweiligen Namen. Dem wirkt Astor allerdings entgegen, indem er - zumindest bei den nicht-deutschen Namen - auf Weltstars zurückgreift.

Wenn der Nachname durch ein Lexem anderer Wortart ersetzt wird (vgl. z. B. durch Verben wie in Andy Borg oder Jan Josef Liefers, so bleibt zwar meist die segmentelle Struktur erhalten, aber die suprasegmentelle Ebene erfährt eine Veränderung. Wenn, wie in diesen Fällen, der Nachname zu einer homophonen Verbform umgedeutet wird, verlagert sich der Akzent auf den Vornamen. Insofern sind hier die segmentellen Homophone am häufigsten.

Als besonders komplexe lexikalische Transformation stellt sich die lexikalische Reorganisation dar, die allerdings hier nur entweder den Vor- oder den

5 Die Gesamtzahl von 56 kommt dadurch zustande, dass Vor- und Nachname, soweit beide einbezogen werden, unterschiedliche Wortarten annehmen können, z. B. Kai Pflaume (Pronomen + Substantiv). 
Nachnamen einschließt (Morgan Freeman > morgen früh mähn; Dépardieu > Depp adieu; Alice Schwarzer > All is schwarzer). Angesichts einer Rekombination des Namens in mehrere Lexeme sinkt der Grad der phonetischen Ähnlichkeit weiter bis hin zur Heterophonie (z. B. André Diarrhoe). Diese Variante stellt die größten Anforderungen an die Dekodierungsfähigkeit des Publikums, zum einen, weil der Vorname als cue fehlt, zum anderen, weil mehr als ein Eintrag im mentalen Lexikon gesucht werden muss. Insofern verwundert es nicht, dass hier die Homoiophonie die häufigste Variante bildet. Wie weiter oben dargelegt, beansprucht diese erschwerte Aufgabe auch mehr Zeit zur Dekodierung als die einfacheren.

\section{Signale an das Publikum}

Angesichts der mit seinem dichten Spiel mit den Namen Prominenter verbundenen kognitiven Herausforderungen überlässt Willy Astor das Gelingen der Pointen nicht dem Zufall. Er verwendet eine breite Palette lexikalischer und phonetischer Hinweise an das Publikum, die der Signalisierung der jeweiligen Pointe dienen. Bereits beim ersten „Mitbewohner“ werden die Zuhörer mit der lexikalischen Umdeutung von Vor- und Nachname konfrontiert (Kai Pflaume), wobei dieses Wortspiel gleich durch mehrere Mechanismen vorbereitet wird (s. u.). Auch in der Folge werden die Hinweise an das Publikum besonders zahlreich verwendet, wenn ein Themenwechsel stattfindet (z. B. von den Charakteren der Mitbewohner zur Gartenpflege und dann zur Politik). Diese Interpretationshilfen werden im Folgenden detaillierter vorgestellt.

\subsection{Lexikalische Mittel}

Lexikalische Mittel können in unterschiedlicher Weise verwendet werden, um das Publikum für eine bevorstehende Pointe zu sensibilisieren. Astor arbeitet hier hauptsächlich mit Antonymen und Wortfeldern des umgedeuteten Namens. Der erste Promi-Name (Kai/kei Pflaume) wird durch den antonymischen Ausdruck „nur gute Typen“ sowie durch eine relativ lange (Atem-) Pause von über $500 \mathrm{~ms}$ Dauer sehr gut vorbereitet.

Häufiger ist die Einbeziehung von Wortfeldern: Der Name Johann Lafer wird durch die mehrfache Erwähnung des Lexems „Koch“ gebahnt; Liam Neeson ['ni:sən] durch ein vorangestelltes „hatschi“, und der Begriff „Spieleabend“ be- 
reitet auf Günther-Maria Halmer ['halme] vor. Weitere Beispiele ließen sich nennen.

\subsection{Pausen und Tempo}

Durch Pausen und/oder Tempowechsel wird signalisiert, dass etwas Wichtiges bevorsteht bzw. gerade artikuliert wird, und die Aufmerksamkeit des Publikums wird auf die Pointe gelenkt. Pausen können dabei unterschiedliche Ausprägungen annehmen: Stille Pausen bedeuten einfach eine deutlich hörbare Unterbrechung des Redeflusses um mindestens 200 ms und erregen damit die Aufmerksamkeit der Zuhörer. Atempausen sind in der Regel deutlich länger und beinhalten nicht zuletzt durch das Atemgeräusch ein deutlicheres Signal an die Rezipienten. Gefüllte Pausen enthalten zusätzlich einen oder mehrere Häsitationslaute (äh, äm etc.) und bilden damit noch stärker ausgeprägte Hinweise auf das Folgende. ${ }^{6}$ Diese Pausenformen können auch in Kombination auftreten, wie das folgende Beispiel zeigt: „[...] und als ich im Gang <I> äh den John traf wollta $<\mathrm{P}>$ mitkommen". ${ }^{7}$ Das Beispiel zeigt auch, dass Pausen nicht nur vor, sondern auch nach der Pointe gesetzt werden können, um den Zuhörern mehr Zeit zu geben, diese als solche zu erkennen.

Auch punktuelle Verzögerungen des Sprechtempos verlangsamen den Redefluss gleichsam und motivieren das Publikum, innezuhalten und auf den Inhalt besonders konzentriert zu achten. Ein Beispiel ist das Kompositum „Stein-Maja“ (Steinmeier), das sehr langsam und überdeutlich artikuliert wird.

Der Unterschied zwischen Pausen und verlangsamtem Sprechtempo besteht darin, dass erstere (meist) auf die Pointe vorausweisen oder (seltener) rückwirkend eingesetzt werden, während die Tempoveränderung die Pointe selbst betrifft.

\subsection{Tags}

Unter tags werden hier Partikeln wie gell und ne zusammengefasst. Sie unterbrechen den Redefluss und lenken den Fokus auf das Nachfolgende. Ein Beispiel ist folgende Sequenz: „[...] und eins war klar, $<\mathrm{P}>$ ne: $\langle\mathrm{P}>$ Wenn ich mit der Jessica

6 Hierbei wird davon ausgegangen, dass gefüllte Pausen im Kabarett - anders als in der Alltagssprache - bewusst gesetzte Signale an die Zuhörer und keine Zeugnisse der kognitiven Planungstätigkeit des Sprechers darstellen.

$7<$ P > steht für eine deutlich hörbare Pause; $<$ I $>$ steht für eine Atempause. 
alber, $\langle\mathrm{P}>$ ne, $\langle\mathrm{P}>$ dann brauch ich mich hinterher nimmer bei der Lennifer einnisten." Das Beispiel veranschaulicht ebenfalls das Zusammenspiel unterschiedlicher Mechanismen, hier tags und Pausen.

\subsection{Kataphorische Nennung von Namensbestandteilen}

Der am einfachsten zu realisierende Hinweisreiz auf eine konkrete Person ist zweifellos die kataphorische Nennung des Vornamens. Hierdurch wird Kontext geschaffen, so dass die Dekodierung des Nachnamens quasi vorgebahnt wird (priming). Hiervon macht Astor reichlich Gebrauch, ggf. unter Hinzufügung weiterer cues wie z. B. der Nennung des Berufes wie in „Und der beste Koch bei uns, das muss ich wirklich zugeben, das war der Johann. Der Johann war ein so guter Koch, ehrlich, der hat das auch gelernt, ne, und wir haben alle gedacht, der Johann, der hat eine Freundin. Hat aber gar net gstimmt, der Johann hatte einen Lafer.“

\subsection{Wiederholungen der Pointe}

Die Wiederholung einer Pointe kann als absolute „Notlösung“ in solchen Fällen gelten, in denen das Publikum sie nicht auf Anhieb begreift. Willy Astor greift in einem Fall zu diesem Mittel: Als das Publikum auf das Spiel mit dem Namen Meg Ryan [mega'reIn] („Meckereien“) nicht reagiert, wiederholt Willy Astor diese Pointe kurzerhand, allerdings auch relativ (gemessen an den Lachern) erfolglos. Dies überrascht auf den ersten Blick, da die segmentellen Abweichungen vom

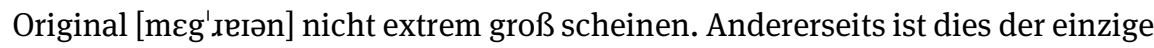
Fall im gesamten Sketch, bei dem die Silbenstruktur beider Namensbestandteile verändert wurde. Dies hat möglicherweise zu den Verständnisschwierigkeiten beigetragen.

\section{Fazit}

Kerngedanke der empirischen Pilotstudie war die Überlegung, dass komplexere Verarbeitungsprozesse seitens der Zuhörer längere Zeit beanspruchen, und dass sich diese durch Messung des Intervalls zwischen der Pointe und dem Erreichen des Lautstärkemaximums der Lacher und - indirekt - durch die Dauer der Lacher messen lässt.

Es wurde ferner angenommen, dass eine Einbeziehung von Vor- und Nachnamen in das Wortspiel schwieriger zu verarbeiten ist als die eines Namens- 
bestandteils. Was die Re-Interpretation der Namen betrifft, so wurde vermutet, dass eine Relexikalisierung (Aufspaltung in mehrere Lexeme) eines Namensbestandteils eine längere Verarbeitungszeit erfordert als eine Umdeutung in ein Substantiv bzw. eine andere Wortart. Beim durchschnittlichen Pegel der Lacher wurde hingegen kein Unterschied erwartet.

Es konnten nicht alle Hypothesen statistisch signifikant bestätigt werden. Auch diejenigen, die das vorgegebene Signifikanzniveau nicht erreicht haben, wurden jedoch zumindest der Tendenz nach bestätigt (H2, H4, H5). Offenbar bindet die zusätzliche Verarbeitung der Vornamen bei gleichzeitiger Abwesenheit des priming effects durch die Vornamen „Rechenkapazität“ auf Seiten der Zuhörer. Dadurch erklärt sich die längere Reaktionszeit. Weiterhin zeigt sich ein Trend dahingehend, dass die Latenzzeiten der Lacher kürzer sind, wenn die Namen zu Substantiven umgedeutet werden verglichen mit den Fällen, in denen mehrere Lexeme aufgespalten werden.

Als Erklärung für die Ergebnisse, die keine Signifikanz erreicht haben, können folgende Faktoren herangezogen werden:

- Die teilweise geringen absoluten Zahlen. (Auswertbar waren lediglich acht Fälle von Vor- und Nachnamen). Dies ist durch die Struktur des Sketches vorgegeben und somit nicht vermehrbar.

- Pragmatische Erfordernisse der Bühnenpräsenz. Der Künstler wartet nicht unbedingt das Ende der Lacher ab, sondern unterbricht sie zum Teil, um die Bühnenpräsenz aufrecht zu erhalten. Insofern bildet die Dauer der Lacher vielleicht keinen ideal geeigneten Parameter. - Entsprechend der Erwartung bildet die durchschnittliche Intensität keinen Parameter, mit dessen Hilfe sich die Reaktionen auf die unterschiedlichen lexikalischen Transformationen erklären lassen. Die hier gemachten Beobachtungen stützen jedenfalls die kognitionspsychologischen Annahmen zur unterschiedlichen Verarbeitung von Eigennamen und Substantiven.

Betrachtet man die lexikalische und phonetische Klassifizierung der Wortspiele, so bilden sich deutliche Muster heraus: Werden die Namen in Substantive umgedeutet, so handelt es sich in den meisten Fällen um komplette Homophone, werden sie in eine andere Wortart umgedeutet, so geht damit eine Restrukturierung der suprasegmentellen Ebene einher. Die Aufteilung in mehrere Lexeme bleibt in den meisten Fällen immer noch sehr nah an der ursprünglichen Lautung und behält vor allem die Anzahl der Silben sowie die Abfolge betonter und unbetonter Silben bei. Der einzige Fall von Diskrepanzen hinsichtlich der Silbenzahl führt zum „Flop“ der Pointe. Schließlich konnte festgestellt werden, 
dass der Autor eine breite Palette von Möglichkeiten nutzt, um das Publikum auf die einzelnen Pointen vorzubereiten.

\section{Literaturangaben}

Braun, Angelika. 2018. Approaching wordplay from the angle of phonology and phonetics examples from German. In Sabine Arndt-Lappe, Angelika Braun, Claudine Moulin \& Esme Winter-Froemel (eds.), Expanding the Lexicon. Linguistic Innovation, Morphological Productivity, and Ludicity (The Dynamics of Wordplay 5), 173-202. Berlin \& Boston: De Gruyter.

Brédart, Serge, Tim Brennen \& Tim Valentine. 1997. Dissociations between the Processing of Proper and Common Names. Cognitive Neuropsychology 14(2). 209-217.

Hollis, Jarrod \& Tim Valentine. 2001. Proper-Name Processing: Are Proper Names Pure Referencing Expressions? Journal of Experimental Psychology: Learning, Memory, and Cognition 27(1). 99-116.

Proverbio, Alice Mado, Serena Mariani, Alberto Zani \& Roberta Adorni. 2009. How are 'Barack Obama' and 'President Elect' Differentially Stored in the Brain? An ERP Investigation on the Processing of Proper and Common Noun Pairs. PLoS ONE 4(9). e7126.

Semenza, Carlo. 2009. The Neurophysiology of Proper Names. Mind \& Language 24(4). 347369.

Valentine, Tim, Viv Moore \& Serge Brédart. 1995. Priming Production of People's Names. The Quarterly Journal of Experimental Psychology 48A(3). 513-535.

Angelika Braun studierte Germanistische Linguistik und Phonetik an der Universität Marburg und wurde dort im Jahr 1988 promoviert. Von 1986 bis 2000 war sie als forensisch-phonetische Sachverständige am Bundeskriminalamt und am Landeskriminalamt Nordrhein-Westfalen tätig. Im Juli 2000 habilitierte sie sich an der Universität Marburg mit der venia legendi für Phonetik und Sprachverarbeitung. Im selben Jahr kehrte sie an die Universität Marburg zurück. Seit 2009 ist sie als Professorin für Allgemeine und Angewandte Phonetik an der Universität Trier tätig. Ihre Forschungsinteressen gelten Sprechermerkmalen sowie soziophonetischen Themen wie Sprache und Emotion, uneigentlichem Sprechen (Ironie), aber auch phonetischen Aspekten des Wortspiels. Während ihrer gesamten akademischen Karriere hat sie sich außerdem intensiv mit der Geschichte der Phonetik beschäftigt. 


\section{Anhang: Verschriftung des Sketches Promi-WG von Willy Astor}

Quelle: https://www.youtube.com/watch?v=UD_mdj7zkdE (letzter Zugriff am 17.09.2018)

Für die Verschriftung gelten folgende typographische Konventionen:

- betonte Silben sind unterstrichen, sofern das Muster vom Hauptakzent im Nachnamen abweicht;

- Häsitationen sind mit <äh> gekennzeichnet; ggf. verbunden mit dem vorausgehenden Wort;

- $\quad$ Deutlich hörbare Atempausen sind mit <I> gekennzeichnet;

- Längere Pausen sind mit $<\mathrm{P}>$ gekennzeichnet;

- Zur Erleichterung des Mitlesens sind regionalsprachliche Charakteristika nach Möglichkeit durch die Schreibung repräsentiert.

Was die wenigsten wissen, ich hab tatsächlich vor $<$ I $>$ dreißig Jahren mal $<$ P $>$ vier Monate in Berlin gwohnt in einer WG, das war a riesen Altbauwohnung in Schöneberg $<$ I $>$ undääh warn $<\mathrm{P}>$ damals auch schon paar Prominente $<\mathrm{P}>$ mit dabei und $\langle$ P $>$ mir ham halt gsagt, bei uns dürfen nur gute $<$ P $>$ Typen wohnen $<$ I $>$ kei Pflaume [Kai Pflaume].

Drogen gab's bei uns natürlich keine, $<\mathrm{P}>$ gut, sicherlich ab und zu brachte die Wencke Myrrhe [Wencke Myhre].

Wir verreisten gerne, also einmal zum Beispiel fuhr ich mit dem Harrison fort [Harrison Ford], und als ich im Gang $<\mathrm{I}>$ äh den Lohn traf wollta $<\mathrm{P}>$ [John Travolta] mitkommen.

Der Anton aus Tirol fragte mich oft, ob ich ein französisches Auto hab, sag ich: „Anton, i hob kins“ [Anthony Hopkins]. Aber soweit ich weiß, fährt der Jean Renault [Jean Reno].

Ich weiß noch, einmal kam ich nach Hause, völlig fertig van der Fahrt [van der Vaart]. Ich war gerade eingeschlafen, plötzlich hörte ich: „Hatschi!“ <P> Plötzlich hörte ich Liam niesen [Liam Neeson]. Der wusste ja nicht, dass ich schon penn [Sean Penn].

Spieleabend war bei uns sehr beliebt, also ab und zu spielte ich mit dem Günther Maria Halma [Günther Maria Halmer] oder schaute mal $\langle\mathrm{P}\rangle$ mit der Cameron Dias [Cameron Diaz].

Um Mitternacht oft noch eine Partie Schach mit der Frau Paltrow. $<$ P $>$ Bevors losging, sagte sie: $<\mathrm{P}>$ „Ich g’winn es“! [Gwyneth Paltrow]. 
Dazu bestellten wir uns eine Pizza beim J J, $<\mathrm{P}>$ also beim $<\mathrm{P}>$ Jan Josef. Ich rief nur an und sagte: Jan Цosef! $<\mathrm{P}>$ Liefers! [Jan-Josef Liefers].

Die Pizza war ja immer so groß, gell, $<$ I $>$ also äh $<$ P $>$ ich aß die ja nie auf, $<$ I $>$ aber der Bruno ganz [Bruno Ganz].

Meistens kochten wir, $\langle\mathrm{P}\rangle$ stand da auf einer Tafel drauf, gell, so zum Beispiel: $\langle\mathrm{P}\rangle$ „Heute brät Pitt“ [Brad Pitt]. Und der beste $\langle\mathrm{P}\rangle$ Koch bei uns, das muss ich wirklich zugeben, das war der Johann. $\langle\mathrm{P}\rangle$ Der Johann war ein so $\langle\mathrm{P}\rangle$ guter Koch, $\langle\mathrm{P}\rangle$ ehrlich, der hat das auch gelernt, ne, $\langle\mathrm{I}>$ und wir haben alle gedacht, der Johann, $<$ I $>$ der hat eine Freundin. $<$ P $>$ Hat aber gar net gstimmt, $<$ I $>$ der Johann hatte einen lafer [Johann Lafer].

Also richtig aufgregt hab ich mich eigentlich nur über drei Typen bei uns, $<\mathrm{I}>$ und zwar, weil ich zum Beispiel einmal sah, wie mir $<\mathrm{I}>$ meinen Erdbeerjoghurt der Armin Müller stahl [Armin Müller-Stahl].

Ja undäh, $<\mathrm{P}>$ weißt Du, der Kühlschrank war sowieso unser begehrtestes Möbelstück, die Melanie wusste sofort, wo der Griff is [Melanie Griffith], und eins war klar:

Wenn der Karl in die Speisekammer geht, $<\mathrm{P}>$ ist klar, dass hinterher wieder was aus dem Lager fehlt [Karl Lagerfeld].

Am meisten nervte mich aber ehrlich gsagt der George, $<\mathrm{P}>$ der putzte das Klo nie [George Clooney].

Weißt, der hörte $<$ P $>$ auch immer heimlich $<$ P $>$ Wiener Walzer $<$ P $>$ auf dem Klo und bekam dann von diesem André Diarrhö [André Rieu].

Weißt, es gab aber im Grunde keinen, met dem man [Matt Damon] Probleme hatte, äh.

Es gab wenig Meckereien [Meg Ryan], $\langle\mathrm{P}\rangle$ es gab wenig Meckereien, $<\mathrm{I}\rangle$ obwohl die Charaktere ja verschieden warn.

Schau mal, $<$ I $>$ der Niki, $<$ P $>$ ja,$<$ P $>$ der war lauter [Niki Lauda]. $<$ I $>$ Der Ben stiller [Ben Stiller].

Gut, sicher, der Til $<\mathrm{P}>$ war der große Schweiger [Til Schweiger].

Dass der Jürgen an Vogel [Jürgen Vogel] hat, war klar. Und dass der Johnny a Depp [Johnny Depp] war, auch. Und als der dann auszog, $<\mathrm{P}>$ sag ich sogar noch zu dem Depp Adieu [Dépardieu]. Obwohl er mir nicht so bescheuert wie der Charly schien [Charly Sheen].

Der Claus Theo war unser Gärtner [Claus Theo Gärtner]. Sie, der war so faul! $<$ P $>$ Der war so faul, einmal sagte er: $<\mathrm{I}>$,Also die Balkonblumen vom Gregor gieß i [Gregor Gysi], aber den Rasen, den schaff ich heut nimmer gell, $<\mathrm{P}>$ den könnt ich doch auch morgen früh mähn [Morgan Freeman]“.

Ein Stockwerk über uns wohnten ja die Politikstudenten $<\mathrm{I}>$ und äh, $<\mathrm{I}>$ ist jetzt vielleicht nur ganz am Rande, was ganz was ba- $<\mathrm{I}>$ äh-nales [Nahles], $<\mathrm{P}>$ 
und zwar, äh, $<\mathrm{P}>$ die hatten nämlich auf dem Balkon eine Biene Maja aus Granit, $<$ P $>$ gell, $<$ I $>$ und äh drum ös die mir [Cem Özdemir] des aufgefallen $<$ I $>$ und äh $<\mathrm{P}>$ da sagt $\mathrm{i}$, „Was machts ihr denn mit einer Biene aus Granit?“ $<\mathrm{P}>$ Da sagen die: „Ja, das ist unsere Stein Maja“ [Steinmeier]. $<$ P $>$ Und die gibt der Hannelore Kraft [Hannelore Kraft].

Und da hams a no $<\mathrm{P}>$ hams a no so an, $<\mathrm{P}>$ an Adler hams ghabt als Haustier, gell, den hams Seehofer genannt $<\mathrm{I}>$ und und der war so deppert, gell, der der ist am Abend ist er nie, hat er nie zurückgefunden in seinen Horst [Horst Seehofer]. Kurz $<\mathrm{I}>$ vielleicht noch $\langle\mathrm{P}\rangle$ zu unseren Frauen, also $<\mathrm{I}>$ die Mädels waren echt okay, $\langle\mathrm{I}>$ also $\langle\mathrm{P}>$ manchmal störten mich beim Schmusen Giselles Bündchen [Giselle Bündchen], und eins war klar, $<\mathrm{P}>$ ne: $<\mathrm{P}>$ Wenn ich mit der Lessica alber [Jessica Alba], $\langle\mathrm{P}\rangle$ ne, $\langle\mathrm{P}\rangle$ dann brauch ich mich hinterher nimmer bei der Lennifer einnisten [Jennifer Aniston].

Ich hab auch öfter in der Badewanne, hab ich auch öfter mit der Kate gepläntschet [Kate Blanchett].

Wir saßen dann oft unter meiner Decke, und da sagte sie: „Mensch, hier ist aber ganz schön dunkel. $<\mathrm{I}>$ “ Sag ich: „Ja, $<\mathrm{P}>$ nur im All is schwarzer [Alice Schwarzer].

Einmal klopfte Frau Fischer bei mir, sag ich: „Komm rein, mir gefällt deine Birne, Helene [Helene Fischer], wenn du willst, zeig ich dir mein Silbereisen [(Florian) Silbereisen].“

Also, am $\langle$ P $>$ am schönsten war's bei uns an Weihnachten, gell, weil wir spielten ja die Weihnachtsgeschichte selber nach, gell. <I> Und der Uwe, der war immer der Ochsenknecht [Uwe Ochsenknecht].

Und und der Robert zum Beispiel, $<\mathrm{I}>$ der wollt immer das Nikolausgedicht aufsagen, aber der war so nervös, der, $<\mathrm{I}>$ der hat das nie $<\mathrm{P}>$ ganz gschafft, der hat immer gsagt: $\langle\mathrm{I}>$, Von drauß vom Walde komm, $<\mathrm{I}>$ ähm, $<\mathrm{P}>$ von drauß vom Walde komm $<\mathrm{P}\rangle$ “ Un mir ham immer gsagt: „Robert, $<\mathrm{P}>$ red fort [Robert Redford]"!

Ich war ja wirklich oft pleite, $\langle\mathrm{P}>$ gell in der $\langle\mathrm{P}>$ ich hatte nie Geld, in der Hoffnung, dass ich amal was vom Daniel kreig [Daniel Craig].

Oder klopfte bei meinem Zimmernachbarn und hab gsagt: $\langle\mathrm{P}\rangle$, ,Hast du vielleicht bissl an Kies, Richard [Keith Richards]? Weil ich mir sonst was vom Andy borg [Andy Borg].

„Nein“, sagt er, „geh amal lieber zur Ursula, kannst dir auch mal was von der leihen [Ursula von der Leyen]“. 
